\title{
2+1 flavor light hadron spectrum and quark masses with the $O(a)$ improved Wilson-clover quark formalism
}

\section{CP-PACS and JLQCD Collaborations:}

T. Ishikawa* ${ }^{\dagger} a$, S. Aoki $^{a, b}$, M. Fukugita $^{c}$, S. Hashimoto ${ }^{d, e}$, K.-I. Ishikawa ${ }^{f}$, N. Ishizuka ${ }^{a, g}$, Y. Iwasaki ${ }^{a}$, K. Kanaya ${ }^{a}$, T. Kaneko ${ }^{d}, e$, Y. Kuramashi ${ }^{a, g}$, M. Okawa ${ }^{f}$, Y. Taniguchi ${ }^{a, g}$, N. Tsutsui ${ }^{d}$, A. Ukawa ${ }^{a, g}$, N. Yamada ${ }^{d, e}$ and T. Yoshié ${ }^{a, g}$

${ }^{a}$ Graduate School of Pure and Applied Sciences, University of Tsukuba, Tsukuba, Ibaraki 305-8571, Japan

${ }^{b}$ Riken BNL Research Center, Brookhaven National Laboratory, Upton, New York 11973, USA

${ }^{c}$ Institute for Cosmic Ray Research, University of Tokyo, Kashiwa 277-8582, Japan

${ }^{d}$ High Energy Accelerator Research Organization (KEK), Tsukuba 305-0801, Japan

e School of High Energy Accelerator Science, The Graduate University for Advanced Studies (Sokendai), Tsukuba 305-0801, Japan

${ }^{f}$ Department of Physics, Hiroshima University, Higashi-Hiroshima, Hiroshima 739-8526, Japan

${ }^{g}$ Center for Computational Sciences, University of Tsukuba, Tsukuba, Ibaraki 305-8577, Japan

We present a summary of results of the joint CP-PACS and JLQCD project toward a $2+1$ flavor full QCD simulation with the $O(a)$-improved Wilson quark formalism and the Iwasaki gauge action. Configurations were generated during 2002-2005 at three lattice spacings, $a \sim 0.076,0.100$ and $0.122 \mathrm{fm}$, keeping the physical volume constant at $(2.0 \mathrm{fm})^{3}$. Up and down quark masses are taken in the range $m_{P S} / m_{V} \sim 0.6-0.78$. We have completed the analysis for the light meson spectrum and quark masses in the continuum limit using the full configuration set. The predicted meson masses reproduce experimental values in the continuum limit at a $1 \%$ level. The average up and down, and strange quark masses turn out to be $m_{u d}^{\overline{\mathrm{MS}}}(\mu=2 \mathrm{GeV})=3.50(14)\left({ }_{-15}^{+26}\right) \mathrm{MeV}$ and $m_{s} \overline{\mathrm{MS}}(\mu=2 \mathrm{GeV})=91.8(3.9)\left({ }_{-4.1}^{+6.8}\right) \mathrm{MeV}$. We discuss our future strategy toward definitive results on hadron spectroscopy with the Wilson-clover formalism.

XXIV International Symposium on Lattice Field Theory

July 23-28 2006

Tucson Arizona, US

\footnotetext{
${ }^{*}$ Speaker.

†E-mail: tomomi@ccs.tsukuba.ac.jp
} 


\section{Introduction}

The calculation of the light hadron spectrum and quark masses is a fundamental and necessary step for the entire area of lattice QCD simulations. Moving from a precision quenched calculation [1] to two flavor $\left(N_{f}=2\right)$ full QCD [2], we observed a significant dynamical up and down ("light") quark effect, which removes most of the $\mathrm{O}(10 \%)$ systematic deviation in the quenched QCD spectrum from experiment. It was also found that light quark masses are significantly reduced in $N_{f}=2$ full QCD. In order to remove the quenching error of the heavier strange quark, the CP-PACS and JLQCD collaborations have jointly pursued a $2+1$ flavor $\left(N_{f}=2+1\right)$ full QCD simulation [3] since 2001. We employ the Wilson quark formalism as in our quenched and $N_{f}=2$ studies, preferring an unambiguous quark-flavor interpretation over the computational ease of the staggered formalism carried out by the MILC collaboration [4].

The project explored the light quark mass range corresponding to $m_{\pi} / m_{\rho}=0.6-0.78$ for which the configuration generation has been completed at three lattice spacings in the fall of 2005 . In this article, we present a summary of the results for light meson masses, light quark masses, pseudoscalar (PS) decay constants and the Sommer scale, evaluated in the continuum limit using the full set of configurations. We also discuss systematic error from chiral extrapolations by comparing fits with polynomial functions of quark masses and those based on chiral perturbation theory $(\chi \mathrm{PT})$.

\section{Production of the gauge configuration}

For the lattice action, we employ the renormalization group (RG) improved Iwasaki gauge action and the clover quark action with the improvement coefficient $c_{S W}$ determined non-perturbatively for the RG action [5]. The choice of the gauge action is made to avoid a first-order phase transition (lattice artifact) observed for the plaquette gauge action [6].

Configurations are generated with the Polynomial Hybrid Monte Carlo (PHMC) algorithm. (See [7] for our implementation.) The molecular dynamics time step $\delta \tau$ and the polynomial order $N_{\text {poly }}$ are chosen such that the HMC and the global Metropolis acceptance rate achieves $85 \%$ and $90 \%$, respectively.

Simulations are performed at three values of the coupling constant such that $a^{2}$ is placed at an even interval. The physical volume is fixed at $(2.0 \mathrm{fm})^{3}$. Main simulation parameters are listed in Table 1. At each coupling, we generate configurations for ten combinations of hopping parameters $\left(\kappa_{u d}, \kappa_{s}\right)$, five for the ud quark mass taken in the range of the pseudoscalar (PS) to vector (V) meson mass ratio of $m_{P S} / m_{V} \sim 0.6-0.78$ and two for the strange quark mass chosen around $m_{P S} / m_{V} \sim 0.7$.

\begin{tabular}{ccccc}
\hline$\beta$ & size & $a[\mathrm{fm}](K$-input $)$ & $a[\mathrm{fm}](\phi$-input $)$ & trajectory \\
\hline 1.83 & $16^{3} \times 32$ & $0.1222(17)$ & $0.1233(20)$ & $7000-8600$ \\
1.90 & $20^{3} \times 40$ & $0.0994(19)$ & $0.0995(19)$ & $5000-9200$ \\
2.05 & $28^{3} \times 56$ & $0.0693(26)$ & $0.0695(26)$ & $6000-6500$ \\
\hline
\end{tabular}

Table 1: Main simulation parameters. 


\section{Measurement and analysis}

Measurements are made at every $10 \mathrm{HMC}$ trajectories. We use the combination of smeared source and point sink, with which the effective masses reach plateau earliest and the statistical errors are smallest. Meson masses and amplitude of the correlator are obtained from single mass $\chi^{2}$ fits to correlators $\langle P(t) P(0)\rangle,\langle V(t) V(0)\rangle$ and $\left\langle A_{4}(t) P(0)\right\rangle$, where $P, V$ and $A_{\mu}$ denote the PS, the vector and the non-perturbatively $O(a)$-improved [8] axial-vector current, respectively. We include correlations in time but ignore those among correlators, since our statistics are not sufficient for the latter. Errors are estimated by the binned jackknife method with the bin size of 100 HMC trajectories.

For chiral extrapolations of meson masses, we use two definitions of the quark mass, the vector Ward identity (VWI) quark mass $m_{q}^{V W I}=\left(1 / \kappa-1 / \kappa_{c}\right) / 2\left(\kappa_{c}\right.$ is the critical hopping parameter where $m_{P S}$ at $\kappa_{u d}=\kappa_{s}=\kappa_{v a l}=\kappa_{c}$ vanishes), and the axial-vector Ward identity (AWI) quark mass $m_{q}^{A W I}=\nabla_{\mu} A_{\mu}(x) /(2 P(x))$. Quark masses are determined from chiral fits with $m_{q}^{A W I}$, because $m_{q}^{V W I}$ shows large scaling violation. ${ }^{1}$ We use chiral fits with $m_{q}^{V W I}$ for other quantities.

Chiral fits are made to light-light, light-strange and strange-strange meson masses simultaneously ignoring their correlations, using a quadratic polynomial function of the sea and valence quark masses;

$$
\begin{aligned}
f\left(\mathrm{M}_{\mathrm{s}}, \mathrm{M}_{\mathrm{v}}\right)= & A+B_{S} \operatorname{trM}_{\mathrm{s}}+B_{V} \operatorname{trM}_{\mathrm{v}}+D_{S V} \operatorname{trM}_{\mathrm{s}} \operatorname{trM}_{\mathrm{v}} \\
& +C_{S 1} \operatorname{trM}_{\mathrm{s}}^{2}+C_{S 2}\left(\operatorname{trM}_{\mathrm{s}}\right)^{2}+C_{V 1} \operatorname{trM}_{\mathrm{v}}^{2}+C_{V 2}\left(\operatorname{trM}_{\mathrm{v}}\right)^{2},
\end{aligned}
$$

where $\mathrm{M}_{\mathrm{S}}=\operatorname{diag}\left(m_{u d}, m_{u d}, m_{s}\right), \mathrm{M}_{\mathrm{V}}=\operatorname{diag}\left(m_{v a l 1}, m_{v a l 2}\right)$, and "tr" means trace of matrices. We set $A=0$ for fits of $m_{P S}^{2}$ with $m_{q}^{V W I}$, while $A=B_{S}=C_{S 1}=C_{S 2}=0$ for those with $m_{q}^{A W I}$.

The physical point is fixed for two cases. The " $K$-input" takes the experimental values $m_{\pi}=$ $0.1350 \mathrm{GeV}, m_{\rho}=0.7684 \mathrm{GeV}$ and $m_{K}=0.4977 \mathrm{GeV}$ as inputs. In the " $\phi$-input" case, $m_{\pi}, m_{\rho}$ and $m_{\phi}=1.0194 \mathrm{GeV}$ are taken as inputs. The lattice spacings determined from the $K$ - and $\phi$ inputs are consistent with each other, as shown in Table 1.

Static potential $V(r)$ is determined from Wilson loops for smeared gauge links and is fitted to a form $V(r)=c-\alpha / r+\sigma r$. The Sommer scale $r_{0}$ calculated from the fit is extrapolated to the physical point as $1 / r_{0}=A+B_{S} \operatorname{tr} \mathrm{M}_{\mathrm{s}}$ using $m_{q}^{V W I}$.

\section{Physics results}

\subsection{Light meson spectrum}

The meson masses are well fitted by linear functions in $a^{2}$, as shown in Fig. 1. We obtain in the continuum limit,

$$
\begin{aligned}
& m_{K^{*}}=0.8961(72) \mathrm{GeV}, \quad m_{\phi}=1.023(14) \mathrm{GeV} \quad(K-\text { input }), \\
& m_{K}=0.495(14) \mathrm{GeV}, \quad m_{K^{*}}=0.8947(12) \mathrm{GeV} \quad(\phi-\text { input }) .
\end{aligned}
$$

\footnotetext{
${ }^{1}$ The VWI quark mass for the ud quarks is negative at our simulation points. This originates from a lack of chiral symmetry of the Wilson quark action. This is another reason to prefer the AWI definition.
} 

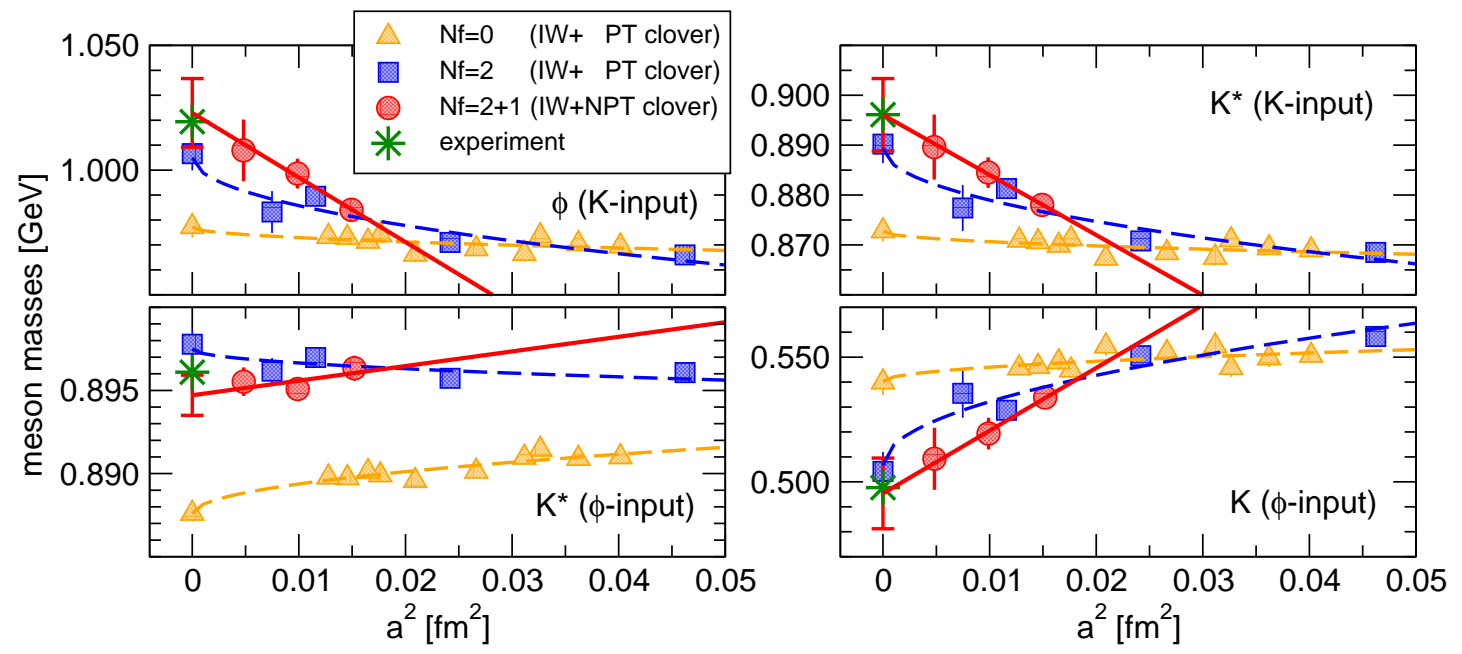

Figure 1: Continuum extrapolation of meson masses, comparing with those for quenched and $N_{f}=2$ QCD [2]. Note that the quenched and $N_{f}=2$ simulations are made with the one-loop perturbatively $O(a)$ improved clover action. Thus extrapolations are made linearly in $a$.

These values are consistent with experiment within the quoted statistical errors of about $1 \%$ level. The errors are larger than those achieved in the $N_{f}=2$ case, and possible deviations from $N_{f}=2$ QCD are not detected. We observe that the scaling violation in $N_{f}=2+1$ QCD seems to be larger than that in the quenched or $N_{f}=2$ case [2] even though the actions used in these cases are less improved than the present $N_{f}=2+1$ case. We may argue, however, that the magnitude of the scaling violation in $N_{f}=2+1 \mathrm{QCD}$ is not particularly large. A quadratic fit of the $K^{*}$ meson mass with $K$-input $m_{K^{*}}=m_{0}\left(1+c\left(\Lambda_{Q C D} \cdot a\right)^{2}\right)$ yields $m_{0}=8960(73) \mathrm{MeV}$ and $c=-1.29(59)$ for $\Lambda_{Q C D}=200 \mathrm{MeV}$. The $\mathrm{O}(1)$ magnitude of the coefficient $c$ is reasonable.

\subsection{Quark masses}

The physical quark mass is determined for the $\overline{\mathrm{MS}}$ scheme at the scale $\mu=2 \mathrm{GeV}$. Lattice results are translated to the $\overline{\mathrm{MS}}$ scheme at $\mu=a^{-1}$ using tadpole-improved one-loop matching factor [9], and then evolved to $\mu=2 \mathrm{GeV}$ using the four-loop RG-equation.

Quark mass results are shown in Fig. 2. While $O\left(g^{4} a\right)$ scaling violation should be present due to the use of one-loop matching factor, comparison of VWI and AWI masses for ud quarks suggests that these terms are small relative to the $O\left(a^{2}\right)$ term [3]. Therefore we extrapolate quark masses linearly in $a^{2}$. Possible effects of $O\left(g^{4} a\right)$ terms are estimated from the ambiguity of the renormalization factor by either shifting the matching scale from $\mu=1 / a$ to $\mu=\pi / a$ or using an alternative definition of coupling for tadpole improvement [2].

As already observed in $N_{f}=2$ QCD [2], values of the strange quark mass determined for either the $K$ - or the $\phi$-inputs, while different at finite lattice spacings, extrapolate to a common value in the continuum limit. Therefore the quark masses in the continuum limit is estimated from a combined fit to data with the $K$ - and the $\phi$-inputs. We finally obtain

$$
m_{u d}^{\overline{\mathrm{MS}}}(\mu=2 \mathrm{GeV})=3.50(14)\left({ }_{-15}^{+26}\right) \mathrm{MeV}, \quad m_{s}^{\overline{\mathrm{MS}}}(\mu=2 \mathrm{GeV})=91.8(3.9)\left({ }_{-4.1}^{+6.8}\right) \mathrm{MeV}
$$



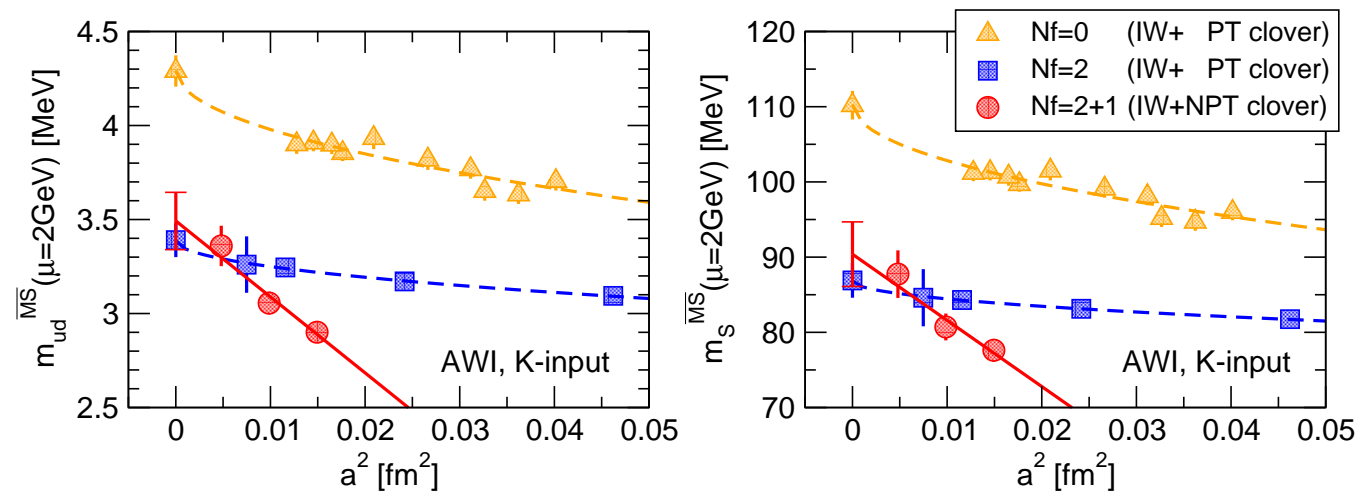

Figure 2: Continuum extrapolations of the up, down and strange quark masses obtained with the $K$-input. For comparison, results for quenched and $N_{f}=2$ QCD [2] are overlaid.

Dynamical up and down quarks reduce significantly the quark masses [2]. The effect of strange quark is less dramatic, and we do not see deviations from the $N_{f}=2$ results beyond statistical errors.

\subsection{PS decay constants}

PS meson decay constants are estimated using matching factor determined by tadpole-improved one-loop perturbation theory. The results with $K$-input are

$$
f_{\pi}=143.4(8.8) \mathrm{GeV}, \quad f_{K}=163.7(8.6) \mathrm{GeV}, \quad f_{K} / f_{\pi}=1.140(17) .
$$

We recall that in our $N_{f}=2$ QCD calculation, the magnitude of scaling violation was so large that we were not able to estimate values in the continuum limit [2]. The situation is much better in the present case and $f_{\pi}$ and $f_{K}$ turn out to be almost consistent with experiment. The errors are large, however. Furthermore, the ratio $f_{K} / f_{\pi}$ differs significantly from experiment. A long chiral extrapolation is a possible cause of the discrepancy.

\subsection{Sommer scale}

The Sommer scale in the continuum limit for $K$-input reads

$$
r_{0}=0.516(21) \mathrm{fm},
$$

which is closer to a phenomenological value of $0.5 \mathrm{fm}$ than the estimate $r_{0} \approx 0.541(17) \mathrm{fm}$ in the $N_{f}=2$ QCD [2].

\section{Chiral fit using the Wilson $\chi \mathbf{P T}$}

Our long chiral extrapolation is a dangerous source of systematic error. As a supplementary analysis, we try to fit meson masses using the $\chi \mathrm{PT}$ and compare it with the polynomial fit above.

We use the $\chi \mathrm{PT}$ modified for the Wilson quark action $(\mathrm{W} \chi \mathrm{PT})[10]$, in which finite lattice spacing corrections to infrared chiral logarithms for the Wilson quark action are incorporated. The 

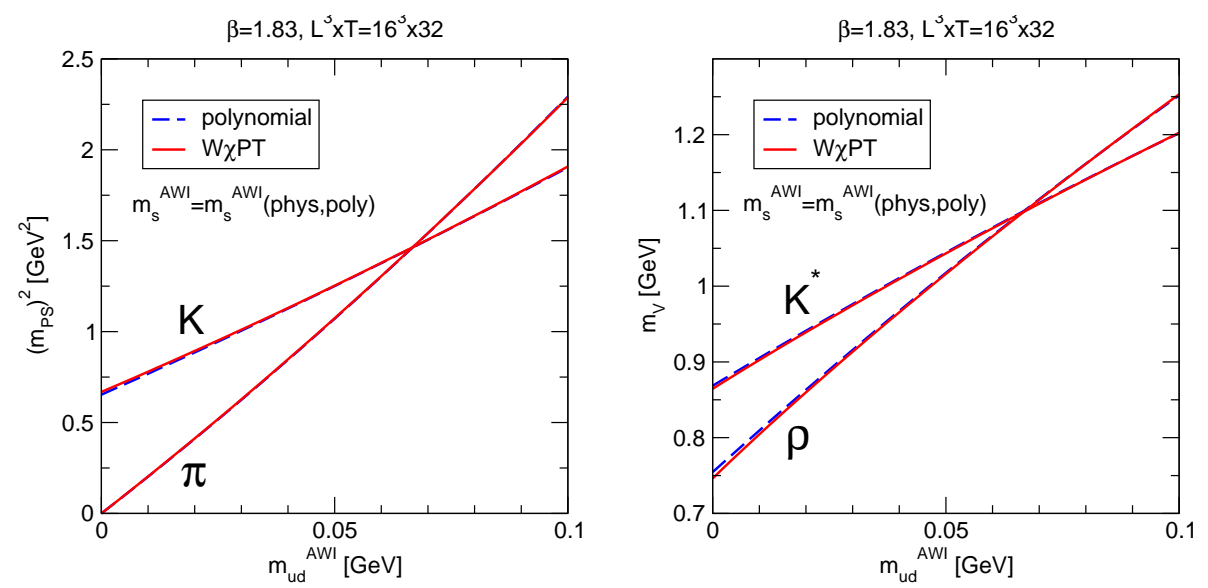

Figure 3: Comparison of the $\mathrm{W} \chi \mathrm{PT}$ fit and the polynomial chiral fits at $\beta=1.83 . m_{s}^{A W I}$ and the lattice spacing necessary to draw curves are set by polynomial chiral fits and the $K$-input.

$N_{f}=2+1 \mathrm{QCD} W \chi \mathrm{PT}$ formulae for the $O(a)$ improved theory has been obtained [11] up to the NLO. For our fit, we rewrite the formulae in terms of the AWI quark mass;

$$
\begin{aligned}
& m_{\theta}^{2}=\left(x+P_{\theta} y\right)\left[1+\frac{1}{f^{2}}\left\{\sum_{\psi=\pi, K, \eta} L_{\psi}(\mu) A_{\psi}^{\theta}-H-12 L_{46}(\mu) x-4 L_{58}(\mu)\left(x+P_{\theta} y\right)\right\}\right], \\
& m_{\omega}=m_{O}+\lambda_{x} x+\lambda_{y} y-\frac{1}{24 \pi^{2} f^{2}} \sum_{\psi=\pi, K, \eta} H_{\psi}^{\omega}\left(g_{1}^{2}, g_{2}^{2}\right)\left(x+P_{\psi} y\right)^{3 / 2}
\end{aligned}
$$

where $\theta=\pi, K$ and $\omega=\rho, K^{*}$. In these equations $P_{\theta}, A_{\psi}^{\theta}$ and $H_{\psi}^{\omega}\left(g_{1}^{2}, g_{2}^{2}\right)$ are geometric factors, $f$ is the pion decay constant in the chiral limit of the LO formula, and

$$
\begin{gathered}
x=\frac{2 B_{0}}{3}\left(2 m_{u d}+m_{s}\right), \quad y=\frac{B_{0}}{3}\left(m_{u d}-m_{s}\right), \\
L_{46}(\mu)=2 L_{4}(\mu)+4 L_{6}(\mu), \quad L_{58}(\mu)=2 L_{5}(\mu)+4 L_{8}(\mu), \\
L_{\psi}(\mu)=\frac{x+P_{\psi} y}{16 \pi^{2}} \ln \frac{x+P_{\psi} y}{\mu^{2}},
\end{gathered}
$$

where $B_{0}, L_{4}, L_{5}, L_{6}$ and $L_{8}$ are the low energy constants in the continuum $\chi$ PT. We note that up to NLO the vector formula has no lattice artifact whereas the PS formula has the term $H$ which is of $O\left(a^{2}\right)$.

We find that while fit parameters vary largely among jackknife samples, and hence are not well determined, fitting curves themselves are stable. Also the $\mathrm{W} \chi \mathrm{PT}$ fitting curves do not exhibit any significant difference from the polynomial fit, as shown in Fig. 3. Therefore, conclusions for the spectrum and quark masses derived from polynomial fits are not altered even if we use the $\mathrm{W} \chi \mathrm{PT}$ fit. We suppose that a relatively large value of the up and down quark masses in our simulation makes it difficult to observe chiral logarithm behavior from data.

\section{Conclusions and future plans}

We have reported the results for the meson spectrum, light quark masses and other physical quantities obtained from our simulations in $N_{f}=2+1$ QCD with Wilson-clover action. We find 
that the meson spectrum is consistent with experiment, and the quark masses are smaller compared to often quoted phenomenological values.

We regard the present result as a first step toward a fully satisfactory $N_{f}=2+1$ simulation. A major point to improve is the control of systematic errors due to quark masses still large compared to those in Nature. Larger volumes will also be needed for baryons, and even for mesons as quark masses are reduced. We hope to overcome these problems with the PACS-CS project [12], using the improved algorithm provided by the domain decomposition idea [13] and the cluster computer PACS-CS developed at University of Tsukuba.

\section{Acknowledgments}

This work is supported by the Epoch Making Simulation Projects of Earth Simulator Center, the Large Scale Simulation Program No.132 (FY2005) of High Energy Accelerator Research Organization (KEK), the Large Scale Simulation Projects of Academic Computing and Communications Center, University of Tsukuba, Inter University Services of Super Computers of Information Technology Center, University of Tokyo, Super Sinet Projects of National Institute of Informatics, and also by the Grant-in-Aid of the Ministry of Education (Nos. 13135216, 13135204, 15540251, 16540228, 16740147, 17340066, 17540259, 17740171, 18104005, 18540250, 18740130, 18740167).

\section{References}

[1] S. Aoki et al. [CP-PACS Collaboration], Phys. Rev. Lett. 84 (2000) 238; Phys. Rev. D67 (2003) 034503.

[2] A. Ali Khan et al. [CP-PACS Collaboration], Phys. Rev. Lett. 85 (2000) 4674, Phys. Rev. Lett. 90 (2003) 029902(E); Phys. Rev. D65 (2002) 054505, Phys. Rev. D67 (2003) 059901(E).

[3] T. Kaneko et al. [CP-PACS and JLQCD Collaborations], Nucl. Phys. B (Proc. Suppl.) 129 (2004) 188; T. Ishikawa et al. [CP-PACS and JLQCD Collaborations], Nucl. Phys. B (Proc. Suppl.) 140 (2005) 225; T. Ishikawa et al. [CP-PACS and JLQCD Collaborations], PoS (LAT2005) 057.

[4] C. Aubin et al. [HPQCD Collaboration, MILC Collaboration and UKQCD Collaboration], Phys. Rev. D70 (2004) 031504(R); Phys. Rev. D70 (2004) 114501.

[5] S. Aoki et al. [CP-PACS Collaboration], Phys. Rev. D73 (2006) 034501.

[6] S. Aoki et al. [JLQCD Collaboration], Phys. Rev. D72 (2005) 054510.

[7] S. Aoki et al. [JLQCD Collaboration], Phys. Rev. D65 (2002) 094507.

[8] T. Kaneko [CP-PACS and JLQCD Collaborations], private communication.

[9] S. Aoki et al., Phys. Rev. D58 (1998) 074505.

[10] S. R. Sharpe and R. L. . Singleton, Phys. Rev. D58 (1998) 074501.

[11] S. Aoki et al., Phys. Rev. D73 (2006) 014511; Phys. Rev. D73 (2006) 094501; S. Takeda, Doctral thesis at Univ. of Tsukuba (2005).

[12] A. Ukawa et al. [PACS-CS Collaboration], PoS (LAT2006) 039; Y. Kuramashi et al. [PACS-CS Collaboration], PoS (LAT2006) 029.

[13] M. Lüscher, JHEP 05 (2003) 052; Comput. Phys. Commun. 156 (2004) 209; Comput. Phys. Commun. 165 (2005) 199. 